\title{
Additive effect of in-hospital TIMI bleeding and chronic kidney disease on 1-year cardiovascular events in patients with acute coronary syndrome
}

\author{
Data from Taiwan Acute Coronary Syndrome Full Spectrum Registry
}

\author{
Tsung-Hsien Lin • Wen-Ter Lai • Chi-Tai Kuo • \\ Juey-Jen Hwang • Fu-Tien Chiang • \\ Shu-Chen Chang $\cdot$ Chee-Jen Chang
}

Received: 20 September 2013/Accepted: 14 March 2014/Published online: 7 May 2014

(C) The Author(s) 2014. This article is published with open access at Springerlink.com

\begin{abstract}
In-hospital bleeding (IHB) is associated with the risk of subsequent cardiovascular events (CVE) in acute coronary syndrome (ACS). We investigated whether increased risk of CVE by IHB is influenced by chronic kidney disease (CKD) or both have detrimental effects on CVE. In a Taiwan national-wide registry, 2819 ACS patients were enrolled. CKD is defined as an estimated glomerular filtration rate of $<60 \mathrm{ml} / \mathrm{min}$ per $1.73 \mathrm{~m}^{2}$. The primary end point is the composite of death, non-fatal myocardial infarction and non-fatal stroke at 12 months. 53 $(1.88 \%)$ and $949(33.7 \%)$ patients suffered from IHB and CKD, respectively. Both IHB and CKD are independently associated with increased risk of the primary end point (HR $2.04,95 \%$ CI $1.05-3.99, p=0.037$ and HR $2.17,95 \%$ CI $1.63-2.87, p<0.01$, respectively). The Kaplan-Meier
\end{abstract}

The manuscript had been partly presented in the 2012 European Society of Cardiology on August 29 in Munich, Germany.

On behalf of ACS Full Spectrum Registry Investigators. Members are listed in the appendix.

T.-H. Lin · W.-T. Lai $(\bowtie)$

Division of Cardiology, Department of Internal Medicine,

Kaohsiung Medical University Hospital, No.100 Tzyou 1st

Road, Kaohsiung 80708, Taiwan, ROC

e-mail: wtlai@cc.kmu.edu.tw

T.-H. Lin · W.-T. Lai

Department of Internal Medicine, Faculty of Medicine,

Kaohsiung Medical University, Kaohsiung, Taiwan

C.-T. Kuo

Chang Gung University College of Medicine, Taoyuan, Taiwan

C.-T. Kuo

Division of Cardiology, Department of Internal Medicine,

Linkou Chang Gung Memorial Hospital, Linkou, Taiwan curves show significantly higher event rates among those with IHB and CKD in the whole, ST-elevation and non-ST elevation populations (all $p<0.01$ ). Patients with $\mathrm{IHB}(+) /$ $\mathrm{CKD}(-)$, $\operatorname{IHB}(-) / \mathrm{CKD}(+)$ and $\operatorname{IHB}(+) / \mathrm{CKD}(+)$ have 1.88 -, 2.13- and 2.98-fold risk to suffer from the primary end point compared with those without IHB and CKD ( $p=0.23,<0.01$ and $<0.01$, respectively). IHB or CKD is independently associated with poor cardiovascular outcome and patients with both IHB and CKD have the worst outcome in ACS.

Keywords Acute coronary syndrome - Chronic kidney disease $\cdot$ Bleeding

$\begin{array}{ll}\text { Abbreviations } \\ \text { CVD } & \text { Cardiovascular disease } \\ \text { ACS } & \text { Acute coronary syndrome } \\ \text { CVE } & \text { Cardiovascular events } \\ \text { PCI } & \text { Percutaneous coronary intervention } \\ \text { CKD } & \text { Chronic kidney disease }\end{array}$

J.-J. Hwang · F.-T. Chiang

Division of Cardiology, Department of Internal Medicine,

National Taiwan University Hospital, Taipei, Taiwan

S.-C. Chang

Division of Biostatistics, Institute of Public Health, National

Yang-Ming University, Taipei, Taiwan

C.-J. Chang

Graduate Institute of Clinical Medicine, Research Center for Clinical Informatics and Medical Statistics, Chang Gung University, Taoyuan, Taiwan 


$\begin{array}{ll}\text { IHB } & \text { In-hospital bleeding } \\ \text { TIMI } & \text { Thrombolysis in myocardial infarction } \\ \text { MI } & \text { Myocardial infarction } \\ \text { GFR } & \text { Glomerular filtration rate } \\ \text { ESRD } & \text { End-stage renal disease } \\ \text { MDRD } & \text { Modification of diet in renal disease } \\ \text { STE-ACS } & \text { ST-elevation acute coronary syndrome } \\ \text { DBP } & \text { Diastolic blood pressure } \\ \text { CAD } & \text { Coronary artery disease } \\ \text { CVA } & \text { Cerebrovascular accident } \\ \text { NSTE-ACS } & \text { Non-ST-elevation acute coronary syndrom }\end{array}$

\section{Introduction}

Cardiovascular disease (CVD) accounts for approximately one-third of all global deaths [1]. The prevalence of CVD has increased considerably in Asian countries over the past several decades as a result of shifts toward a more "westernized" lifestyle. In Taiwan, CVD is the second most common cause of mortality since 2010 [2]. Acute coronary syndrome (ACS) is the most severe form of CVD. Because of its major impact on morbidity and mortality, as well as its contribution to annual health-care costs, it is of the utmost importance to develop improved strategies for reducing cardiovascular events (CVE) and preventing complications.

In ACS, aggressive antiplatelet and anticoagulation therapies have been recently developed and can reduce future CVE, but may increase the risk of bleeding. Anemia and bleeding events have been shown to increase mortality in studies of ACS and percutaneous coronary intervention (PCI) [3, 4]. Because lower body weight could be associated with bleeding complication in ACS, weight-adjusted dose of antithrombotic agent is recommended in the international ACS guidelines [5, 6]. Compared with Caucasians, the Asian population usually has lower body weight and might possibly suffer from antithrombotic and antiplatelet overdose. Although bleeding events increase the risk of mortality in the Caucasian population, no study has been reported in the Asian population.

Chronic kidney disease (CKD) is a risk factor for coronary heart disease and bleeding with antithrombotic therapy in patients with ACS [7, 8]. Whether the association between bleeding and mortality is influenced by the presence of CKD or both have independently detrimental effects on CVE is unknown. In this study, we test the hypothesis that in-hospital bleeding (IHB), using Thrombolysis in Myocardial Infarction (TIMI) bleeding definition, would increase the risk of CVE, and CKD might have an additively detrimental effect on CVE in a prospective cohort in an Asia endemic area of kidney disease [9].

\section{Patients and methods}

Study design

The study was a prospective, national, multicenter, noninterventional, observational design. Patient recruitment and definition of ACS had been previously described in detail [10]. In brief, patients who were aged 20 years or older, who were admitted within $24 \mathrm{~h}$ to the hospital with symptoms of ACS and who provided informed consent were eligible to be included in the study. Patient data, such as baseline characteristics, risk factors, clinical presentation, clinical diagnosis, in-hospital interventions as well as medications prescribed were collected from admission to discharge. Patients were followed up at months 3, 6, 9 and 12 post-discharge and data were collected on medication usage, revascularization strategy as well as clinical events, such as death, myocardial infarction (MI), stroke, revascularization and hospitalization. Monitoring for source documentation and accuracy was performed in $5 \%$ of all case report forms at each recruiting site. This study was carried out in accordance with the local regulatory guidelines and international guidelines for Good Epidemiological Practice [11]. Ethics committee approval was obtained at all trial sites. Written informed consent was given by the patients for their information to be stored in the hospital database and used for research.

Thrombolysis in Myocardial Infarction (TIMI) bleeding classification

TIMI bleeding classification includes major and minor bleeding. TIMI major bleeding is defined as patients with intracranial hemorrhage or $\mathrm{a} \geq 5 \mathrm{~g} / \mathrm{dl}$ decrease in hemoglobin concentration or a $\geq 15 \%$ absolute decrease in hematocrit. If observed with blood loss $\geq 3 \mathrm{~g} / \mathrm{dl}$, decrease in hemoglobin concentration or $\geq 10 \%$ decrease in hematocrit, or no observed blood loss with $\geq 4 \mathrm{~g} / \mathrm{dl}$ decrease in hemoglobin concentration or $\geq 12 \%$ decrease in hematocrit, it is defined as TIMI minor bleeding [12].

\section{Calculation of kidney function and definition of CKD}

The estimated glomerular filtration rate (GFR) was calculated using the Modification of Diet in Renal Disease (MDRD) Study equation [GFR $=186.3 \times$ (serum creatinine in $\mathrm{mg} / \mathrm{dl})^{-1.154} \times(\text { age })^{-0.203} \times(0.742$ if female $\left.)\right]$ [13]. Chronic kidney disease was defined as a GFR $<60 \mathrm{ml} / \mathrm{min}$ per $1.73 \mathrm{~m}^{2}$. This range corresponds to stage 3 or higher CKD by the National Kidney Foundation's classification scheme and helps identify individuals with clinically significant CKD [14]. 
Statistical analysis

The sample size for the Taiwan ACS Full Spectrum Registry was calculated as follows. There are about 50000 new ACS cases per year in Taiwan. Based on a known background incidence rate of 0.0025 , a sample of 2395 patients would achieve $80 \%$ power to detect an additional incidence rate of 0.003 with a precision of $0.2 \%$ and $95 \%$ confidence interval. Taking into account a dropout rate of $20 \%$, a sample of 3000 was considered to be adequately representative.

All data were expressed as mean \pm standard deviation (SD). For comparability between groups, a Chi-square test or Fisher's exact test was used for categorical variables and one-way analysis of variance (ANOVA) was adopted for continuous variables. One-year CVE analysis was performed using Kaplan-Meier survival curves and the logrank test. Univariate and multivariate logistic regression analyses were conducted to analyze odds ratio (OR) and Cox regression model was used for hazard ratio (HR) calculation for IHB or CVE. The adjusted variables in model 1 include age and sex. The adjusted variables in model 2 include model 1 covariates and medicine at discharge (aspirin, clopidogrel, angiotensin-converting enzyme inhibitor, angiotensin II receptor blocker, betablocker and statin). Analyses were conducted as time to first event without double counting of events within analyses involving composite end points.

The primary outcome was the composite CVE of death, non-fatal myocardial infarction and non-fatal stroke at 1 year. The secondary outcome was the CVE of death, non-fatal myocardial infarction, non-fatal stroke, re-hospitalization and revascularization at 1 year. We analyzed the whole, STE-ACS and NSTE-ACS populations separately. Statistical analysis was performed using SAS software version 9.2 (SAS Institute Inc., Cary, NC, USA). All statistical analyses were performed using a level of $<0.05$ with two-sided testing and this was considered as statistically significant.

\section{Results}

\section{Clinical characteristics}

A total of 3183 eligible consecutive patients were enrolled between October 2008 and January 2010 at 39 medical centers and regional hospitals in Taiwan. Among them, $2819(88.6 \%)$ subjects with renal parameters and 12 months outcome data were analyzed in this study and $1537(54.5 \%)$ patients had ST-segment elevation acute coronary syndrome (STE-ACS). The subjects included 2230 men and 589 women (male $79.11 \%$ ). Mean age was $62.9 \pm 13.5$ years.
Overall, $53(1.88 \%)$ patients had TIMI bleeding including $17(0.60 \%)$ major and $36(1.28 \%)$ minor. The TIMI major bleeding included 1 intracranial hemorrhage, 1 coronary artery bypass grafting (CABG)-related bleeding, 8 gastrointestinal (GI) bleeding, 3 genitourinary (GU) bleeding and 5 other location bleeding. The TIMI minor bleeding included 2 vascular access sites bleeding, 5 CABG-related bleeding, 23 GI bleeding, 1 GU bleeding and 4 other location bleeding. Compared with no TIMI bleeding subjects, those with TIMI bleeding were older and thinner, had higher grade of Killip class, lower systolic and diastolic blood pressure (DBP) and MDRD GFR at presentation, and lower percentage of cerebrovascular accident (CVA) (Table 1).

Baseline creatinine was $3.0 \pm 2.6$ and $1.0 \pm 0.2 \mathrm{mg} / \mathrm{dl}$ in the CKD $(n=949)$ and non-CKD $(n=1870)$ groups. Compared with non-CKD subjects, those with CKD were older, shorter and thinner, included more women, had higher grade of Killip class, lower DBP and faster heart rate at presentation. They also had more comorbidity including hypertension, diabetes, previous coronary artery disease (CAD), previous CVA and previous heart failure, but lower percentage of smoking and family history of CAD.

Pharmacological management during admission

Medications prescribed during admission are shown in Table 1. Aspirin, clopidogrel, $\beta$-blocker, statins and unfractional heparin were prescribed less often during admission in patients with than those without TIMI bleeding. Glycoprotein IIb/IIa was prescribed more often to TIMI bleeding patients during admission. There was no significant difference regarding use of low molecular weight heparin, warfarin, ticlopidine and renin angiotensin system blockers between two group.

Binary regression analysis found age [OR 1.03, $95 \%$ confidence interval (CI) 1.01-1.06, $p=0.015$ ], Killip class $(p<0.01)$, use of glycoprotein IIb/IIIa (OR 2.49, $95 \% \mathrm{CI}$ $1.27-4.88, p<0.01$ ) and unfractional heparin (OR 0.36, $95 \%$ CI $0.19-0.68, p<0.01)$ to be independent predictors for occurrence of IHB (Table 2).

\section{Cardiovascular outcomes}

During admission patients with TIMI bleeding had more death and stroke (8.77 vs $1.50 \%$ and 3.51 vs $0.36 \%$, both $p<0.01)$, but similar recurrent myocardial infarction (1.75 vs $0.75 \%, p=0.388$ ) compared with no TIMI bleeding subjects (Table 3). Those with TIMI bleeding still had more death rate at 3, 6, 9 and 12 months follow-up (all $p<0.01$ ). The re-hospitalization rate was higher during the 3 and 6 months follow-up in those suffering from TIMI bleeding (both $p<0.01$ ). 
Table 1 Baseline characteristics between those with and without in-hospital bleeding

\begin{tabular}{|c|c|c|c|c|c|c|c|c|}
\hline $\begin{array}{l}\text { Number }(\%) / \\
\text { mean (SD) }\end{array}$ & $\begin{array}{l}\text { TIMI bleeding } \\
(n=53)\end{array}$ & $\begin{array}{l}\text { No TIMI bleeding } \\
(n=2766)\end{array}$ & $p$ value & $\begin{array}{l}\operatorname{IHB}(-) / \mathrm{CKD}(-) \\
(n=1,846)\end{array}$ & $\begin{array}{l}\mathrm{IHB}(+) / \\
\mathrm{CKD}(-) \\
(n=24)\end{array}$ & $\begin{array}{l}\operatorname{IHB}(-) / \\
\mathrm{CKD}(+) \\
(n=920)\end{array}$ & $\begin{array}{l}\mathrm{IHB}(+) / \\
\mathrm{CKD}(+) \\
(n=29)\end{array}$ & $p$ value \\
\hline Sex (male) & $38(71.70 \%)$ & $2192(79.25 \%)$ & 0.181 & $1530(82.88 \%)$ & $21(87.50 \%)$ & $662(71.96 \%)$ & $17(58.62 \%)$ & $<0.01$ \\
\hline Age (year) & $68.29 \pm 13.83$ & $62.77 \pm 13.51$ & $<0.01$ & $59.45 \pm 13.03$ & $63.30 \pm 15.69$ & $69.43 \pm 11.93$ & $72.42 \pm 10.69$ & $<0.01$ \\
\hline \multicolumn{9}{|l|}{ Killip } \\
\hline Class 1 & $12(26.67 \%)$ & $1394(62.20 \%)$ & $<0.01$ & $1053(69.23 \%)$ & $6(31.58 \%)$ & $341(47.36 \%)$ & $6(23.08 \%)$ & $<0.01$ \\
\hline Class 2 & $8(17.78 \%)$ & $394(17.58 \%)$ & & $262(17.23 \%)$ & $5(26.32 \%)$ & $132(18.33 \%)$ & $3(11.54 \%)$ & \\
\hline Class 3 & $7(15.56 \%)$ & $238(10.62 \%)$ & & $111(7.30 \%)$ & $2(10.53 \%)$ & $127(17.64 \%)$ & $5(19.23 \%)$ & \\
\hline Class 4 & $18(40.00 \%)$ & $215(9.59 \%)$ & & $95(6.25 \%)$ & $6(31.58 \%)$ & $120(16.67 \%)$ & $12(46.15 \%)$ & \\
\hline \multicolumn{9}{|l|}{ Blood pressure } \\
\hline SBP (mmHg) & $126.39 \pm 35.66$ & $139.57 \pm 32.63$ & $<0.01$ & $139.64 \pm 30.47$ & $126.38 \pm 32.98$ & $139.44 \pm 36.63$ & $126.41 \pm 38.51$ & 0.043 \\
\hline DBP (mmHg) & $76.06 \pm 21.67$ & $82.10 \pm 20.82$ & 0.040 & $83.63 \pm 19.73$ & $73.08 \pm 16.82$ & $79.04 \pm 22.55$ & $78.70 \pm 25.25$ & $<0.01$ \\
\hline $\begin{array}{l}\text { Heart rate (beat/ } \\
\text { min) }\end{array}$ & $85.33 \pm 30.80$ & $82.03 \pm 22.13$ & 0.292 & $79.79 \pm 19.66$ & $84.29 \pm 29.36$ & $86.54 \pm 25.84$ & $86.21 \pm 32.50$ & $<0.01$ \\
\hline Height (cm) & $162.13 \pm 7.93$ & $164.03 \pm 7.87$ & 0.082 & $164.60 \pm 7.67$ & $164.67 \pm 6.08$ & $162.90 \pm 8.15$ & $160.03 \pm 8.73$ & $<0.01$ \\
\hline Weight (kg) & $63.98 \pm 12.60$ & $68.70 \pm 12.81$ & $<0.01$ & $69.92 \pm 12.81$ & $66.27 \pm 13.16$ & $66.26 \pm 12.47$ & $62.09 \pm 12.01$ & $<0.01$ \\
\hline $\begin{array}{l}\text { Waist } \\
\text { circumference }\end{array}$ & $86.47 \pm 11.61$ & $90.53 \pm 9.51$ & 0.084 & $90.68 \pm 9.23$ & $83.67 \pm 13.41$ & $90.19 \pm 10.13$ & $88.00 \pm 10.88$ & 0.241 \\
\hline $\begin{array}{l}\text { Creatinine (mg/ } \\
\text { dl) }\end{array}$ & $1.73 \pm 1.43$ & $1.64 \pm 1.82$ & 0.730 & $0.96 \pm 0.19$ & $1.07 \pm 0.16$ & $3.01 \pm 2.65$ & $2.27 \pm 1.76$ & $<0.01$ \\
\hline MDRD eGFR & $56.05 \pm 23.92$ & $73.62 \pm 50.90$ & 0.012 & $92.30 \pm 51.68$ & $77.66 \pm 11.75$ & $36.14 \pm 18.00$ & $38.17 \pm 14.77$ & $<0.01$ \\
\hline Dyslipidemia & $20(37.74 \%)$ & $1073(39.13 \%)$ & 0.837 & $708(38.69 \%)$ & $9(37.50 \%)$ & $365(40.02 \%)$ & $11(37.93 \%)$ & 0.919 \\
\hline Hypertension & $35(66.04 \%)$ & $1741(63.52 \%)$ & 0.706 & $1040(56.83 \%)$ & $13(54.17 \%)$ & $701(76.95 \%)$ & $22(75.86 \%)$ & $<0.01$ \\
\hline Diabetes & $24(45.28 \%)$ & $988(35.91 \%)$ & 0.160 & $505(27.49 \%)$ & $8(33.33 \%)$ & $483(52.84 \%)$ & $16(55.17 \%)$ & $<0.01$ \\
\hline \multicolumn{9}{|l|}{ Smoker } \\
\hline Current & $22(41.51 \%)$ & $1161(42.72 \%)$ & 0.916 & $899(49.48 \%)$ & $15(62.50 \%)$ & $262(29.08 \%)$ & $7(24.14 \%)$ & $<0.01$ \\
\hline Former & $10(18.87 \%)$ & $454(16.70 \%)$ & & $264(14.53 \%)$ & $3(12.50 \%)$ & $190(21.09 \%)$ & $7(24.14 \%)$ & \\
\hline Never & $21(39.62 \%)$ & $1103(40.58 \%)$ & & $654(35.99 \%)$ & $6(25.00 \%)$ & $449(49.83 \%)$ & $15(51.72 \%)$ & \\
\hline $\begin{array}{l}\text { FH of } \\
\text { premature } \\
\text { CAD }\end{array}$ & $7(16.67 \%)$ & $478(22.58 \%)$ & 0.363 & $378(25.93 \%)$ & $5(27.78 \%)$ & $100(15.17 \%)$ & $2(8.33 \%)$ & $<0.01$ \\
\hline Previous CAD & $12(22.64 \%)$ & $663(23.97 \%)$ & 0.822 & $354(19.18 \%)$ & $4(16.67 \%)$ & $309(33.59 \%)$ & $8(27.59 \%)$ & $<0.01$ \\
\hline $\begin{array}{l}\text { Previous heart } \\
\text { failure }\end{array}$ & $4(7.55 \%)$ & $144(5.21 \%)$ & 0.449 & $53(2.87 \%)$ & $1(4.17 \%)$ & $91(9.89 \%)$ & $3(10.34 \%)$ & $<0.01$ \\
\hline Old CVA & $0(0.00 \%)$ & $252(9.11 \%)$ & 0.021 & $118(6.39 \%)$ & $0(0.00 \%)$ & $134(14.57 \%)$ & $0(0.00 \%)$ & $<0.01$ \\
\hline \multicolumn{9}{|c|}{ In-hospital medication } \\
\hline Aspirin & $44(83.02 \%)$ & $2551(92.23 \%)$ & 0.014 & $1738(94.15 \%)$ & $19(79.17 \%)$ & $813(88.37 \%)$ & $25(86.21 \%)$ & $<0.01$ \\
\hline Clopidogrel & $46(86.79 \%)$ & $2614(94.50 \%)$ & 0.016 & $1769(95.83 \%)$ & $21(87.50 \%)$ & $845(91.85 \%)$ & $25(86.21 \%)$ & $<0.01$ \\
\hline Ticlopidine & $0(0.00 \%)$ & $21(0.76 \%)$ & 0.524 & $13(0.70 \%)$ & $0(0.00 \%)$ & $8(0.87 \%)$ & $0(0.00 \%)$ & 0.889 \\
\hline Warfarin & $0(0.00 \%)$ & $27(0.98 \%)$ & 0.470 & $14(0.76 \%)$ & $0(0.00 \%)$ & $13(1.41 \%)$ & $0(0.00 \%)$ & 0.348 \\
\hline $\begin{array}{l}\text { Glycoprotein } \\
\text { IIb/IIIa }\end{array}$ & $17(32.08 \%)$ & $457(16.52 \%)$ & $<0.01$ & $317(17.17 \%)$ & $9(37.50 \%)$ & $140(15.22 \%)$ & $8(27.59 \%)$ & $<0.01$ \\
\hline $\begin{array}{l}\text { Unfractional } \\
\text { heparin }\end{array}$ & $29(54.72 \%)$ & $2024(73.17 \%)$ & $<0.01$ & $1361(73.73 \%)$ & $13(54.17 \%)$ & $663(72.07 \%)$ & $16(55.17 \%)$ & 0.020 \\
\hline LMWH & $16(30.19 \%)$ & $816(29.50 \%)$ & 0.913 & $559(30.28 \%)$ & $9(37.50 \%)$ & $257(27.93 \%)$ & $7(24.14 \%)$ & 0.429 \\
\hline ACEI & $24(45.28 \%)$ & $1392(50.33 \%)$ & 0.467 & $1005(54.44 \%)$ & $10(41.67 \%)$ & $387(42.07 \%)$ & $14(48.28 \%)$ & $<0.01$ \\
\hline ARB & $5(9.43 \%)$ & $316(11.42 \%)$ & 0.651 & $178(9.64 \%)$ & $1(4.17 \%)$ & $138(15.00 \%)$ & $4(13.79 \%)$ & $<0.01$ \\
\hline$\beta$-blocker & $15(28.30 \%)$ & $1268(45.84 \%)$ & 0.011 & $873(47.29 \%)$ & $8(33.33 \%)$ & $395(42.93 \%)$ & $7(24.14 \%)$ & $<0.01$ \\
\hline Statin & $18(33.96 \%)$ & $1367(49.42 \%)$ & 0.026 & $953(51.63 \%)$ & $9(37.50 \%)$ & $414(45.00 \%)$ & $9(31.03 \%)$ & $<0.01$ \\
\hline
\end{tabular}

TIMI thrombolysis in myocardial infarction, $S B P$ systolic blood pressure, $D B P$ diastolic blood pressure, MDRD Modification of Diet in Renal Disease Study, $e G F R$ estimated glomerular filtration rate, $F H$ family history, $C A D$ coronary artery disease, $C V A$ cerebrovascular accident, $L M W H$ low molecular weight heparin, $A C E I$ angiotensin-converting enzyme inhibitor, $A R B$ angiotensin II receptor blocker 
Table 2 Predictors for inhospital TIMI bleeding in binary logistic regression analysis

TIMI Thrombolysis in

Myocardial Infarction, SBP

systolic blood pressure, $D B P$

diastolic blood pressure, $M D R D$

Modification of Diet in Renal

Disease Study, eGFR estimated

glomerular filtration rate

\begin{tabular}{llclc}
\hline & Unadjusted OR $(95 \%$ CI $)$ & $p$ value & Adjusted OR (95 \% CI) & $p$ value \\
\hline Age (year) & $1.03(1.01-1.05)$ & $<0.01$ & $1.03(1.01-1.06)$ & 0.015 \\
Killip IV & 1 & - & 1 & \\
III & $0.35(0.14-0.86)$ & 0.022 & $0.33(0.13-0.83)$ & 0.018 \\
II & $0.24(0.10-0.57)$ & $<0.01$ & $0.25(0.11-0.60)$ & $<0.01$ \\
I & $0.10(0.05-0.22)$ & $<0.01$ & $0.11(0.05-0.24)$ & $<0.01$ \\
SBP & $0.99(0.98-1.00)$ & $<0.01$ & & \\
DBP & $0.99(0.97-1.00)$ & 0.040 & & \\
Weight (kg) & $0.97(0.95-0.99)$ & $<0.01$ & & \\
MDRD eGFR & $0.98(0.98-0.99)$ & $<0.01$ & & $<0.01$ \\
Aspirin & $0.41(0.20-0.86)$ & 0.017 & & $<0.01$ \\
Clopidogrel & $0.38(0.17-0.86)$ & 0.020 & & \\
Glycoprotein IIb/IIIa & $2.39(1.33-4.29)$ & $<0.01$ & $2.49(1.27-4.88)$ & \\
Unfractional heparin & $0.44(0.26-0.77)$ & $<0.01$ & $0.36(0.19-0.68)$ & \\
$\beta$-blocker & $0.47(0.26-0.85)$ & 0.013 & & \\
Statin & $0.53(0.30-0.93)$ & 0.028 & & \\
\hline
\end{tabular}

The unadjusted HR of the presence of TIMI bleeding in the whole, STE-ACS and non-ST-segment elevation ACS (NSTE-ACS) populations were 3.66 (95\% CI 2.18-6.1), 2.88 (95\% CI 1.35-6.18) and 5.36 (95\% CI 2.62-10.95), respectively, for the primary end point. For the secondary end point the HR of the presence of TIMI bleeding in the whole, STE-ACS and NSTE-ACS populations were1.74 (95\% CI 1.19-2.53), 1.35 (95\% CI 0.81-2.25) and 2.58 (95\% CI 1.49-4.49), respectively. The association was statistically significant after adjusting for age, sex and medication at discharge in the NSTE-ACS population for the primary outcomes (HR 2.74, $95 \%$ CI 1.29-5.84, $p<0.01$ ), but not in the STE-ACS population. For the secondary outcome, TIMI bleeding is still a predictor only in the NSTE-ACS population after adjusting for age, sex and medication at discharge (HR $1.95,95 \%$ CI $1.10-3.45, p=0.022)$. There is a trend for inhospital bleeding being a predictor for the primary end point in those with NSTE-ACS after adjusting for age, sex, medication at discharge, creatinine, weight and Killip class (HR 2.34, $95 \%$ CI 0.94-5.86, $p=0.068$ ) (Table 4).

Influence of TIMI bleeding and CKD on cardiovascular outcome

CKD is independently associated with a significant increase of primary end point after adjusting for age, sex and medication at discharge (OR 2.17, $95 \%$ CI 1.63-2.87, $p<0.01)$. The Kaplan-Meier curves show significantly higher primary end point rates among those with IHB and CKD in the whole, STE-ACS and NSTE-ACS populations during 12 months follow-up (all $p<0.01$ ) (Fig. 1). We found an additively detrimental effect on the CVE between TIMI bleeding and CKD on the occurrence of primary end point (Table 5). In patients without CKD, TIMI bleeding had a 1.88-fold risk to have primary end point (HR 1.88, $95 \%$ CI $0.68-5.21 ; p=0.227)$. When patients had no TIMI bleeding, presence of CKD was associated with a 2.13-fold risk of primary endpoint (HR 2.13, $95 \%$ CI 1.62-2.79; $p<0.01$ ), but CKD patients with TIMI bleeding had a 2.98-fold risk for primary endpoint (HR 2.98, $95 \%$ CI 1.55-5.75; $p<0.01)$, compared to the patients without TIMI bleeding and CKD.

\section{Discussion}

There are three major findings in this ACS cohort study. First, patients with IHB had higher risk of in-hospital and 12 months death. Second, IHB is associated with poor cardiovascular outcome, especially in those in the NSTEACS population. Third, patients with both IHB and CKD had the worst prognosis during the 12 months follow-up. Furthermore, they had additively detrimental effect on the cardiovascular outcome.

By using TIMI bleeding definition our study found that ACS patients with IHB had higher risk of in-hospital and 12 months death. Among the different bleeding definitions, TIMI is more capable than ACUITY in identifying patients with bleeding at higher risk for early mortality [15]. However, the other study suggests that bleeding assessed with clinical criteria by Global Use of Strategies to Open Occluded Coronary Arteries (GUSTO) bleeding criteria is more important than that assessed by laboratory criteria with TIMI bleeding criteria in terms of outcomes [16]. Recently, a consensus report from the Bleeding Academic Research Consortium (BARC) proposed standardized bleeding definitions through the use of a hierarchical approach of describing bleeding severity grade in patients 
Table 3 Cumulative

cardiovascular events during index hospitalization, at 3, 6, 9 and 12 months follow-up

\begin{tabular}{|c|c|c|c|c|}
\hline $\begin{array}{l}\text { Parameters number } \\
(\%) / \text { mean } \pm \mathrm{SD}\end{array}$ & $\begin{array}{l}\text { TIMI bleeding } \\
(n=53)(\%)\end{array}$ & $\begin{array}{l}\text { No TIMI bleeding } \\
(n=2766)(\%)\end{array}$ & All $(n=2819)(\%)$ & $p$ value \\
\hline \multicolumn{5}{|l|}{ In-hospital } \\
\hline Death & $5(8.77)$ & $46(1.50)$ & $51(1.63)$ & $<0.01$ \\
\hline Re-infarction & $1(1.75)$ & $23(0.75)$ & $24(0.77)$ & 0.388 \\
\hline Stroke & $2(3.51)$ & $11(0.36)$ & $13(0.42)$ & $<0.01$ \\
\hline \multicolumn{5}{|l|}{ 3-month follow-up } \\
\hline Death & $8(15.69)$ & $84(3.18)$ & $92(3.42)$ & $<0.01$ \\
\hline Myocardial Infarction & $2(.44)$ & $34(1.31)$ & $36(1.37)$ & 0.073 \\
\hline Stroke & $1(2.22)$ & $14(0.54)$ & $15(0.57)$ & 0.137 \\
\hline Re-hospitalization & $14(31.11)$ & $467(18.04)$ & $481(18.27)$ & 0.025 \\
\hline Cardiac & $9(64.29)$ & $311(67.90)$ & $320(67.80)$ & \\
\hline Non-cardiac & $5(35.71)$ & $144(31.44)$ & 149 (31.57) & \\
\hline Both & $0(0.00)$ & $3(0.66)$ & $3(0.64)$ & \\
\hline Unknown & 0 & 9 & 9 & \\
\hline Repeat revascularization & $1(2.22)$ & $55(2.13)$ & $56(2.13)$ & 0.965 \\
\hline \multicolumn{5}{|l|}{ 6-month follow-up } \\
\hline Death & $12(25.53)$ & $117(4.65)$ & $129(5.03)$ & $<0.01$ \\
\hline Myocardial Infarction & $2(5.13)$ & $59(2.44)$ & $61(2.48)$ & 0.284 \\
\hline Stroke & $1(2.63)$ & $24(0.99)$ & $25(1.02)$ & 0.318 \\
\hline Re-hospitalization & $20(47.62)$ & $708(28.51)$ & $728(28.83)$ & $<0.01$ \\
\hline Cardiac & $13(65.00)$ & $483(69.20)$ & $496(69.08)$ & \\
\hline Non-cardiac & $7(35.00)$ & $192(27.51)$ & 199 (27.72) & \\
\hline Both & $0(0.00)$ & $23(3.30)$ & $23(3.20)$ & \\
\hline Unknown & 0 & 10 & 10 & \\
\hline Repeat revascularization & $2(5.26)$ & $89(3.68)$ & $91(3.71)$ & 0.609 \\
\hline \multicolumn{5}{|l|}{ 9-month follow-up } \\
\hline Death & $13(28.26)$ & $135(5.55)$ & $148(5.97)$ & $<0.01$ \\
\hline Myocardial Infarction & $2(5.71)$ & $76(3.28)$ & $78(3.32)$ & 0.425 \\
\hline Stroke & $1(3.03)$ & $30(1.30)$ & $31(1.32)$ & 0.387 \\
\hline Re-hospitalization & $21(51.22)$ & $906(37.59)$ & $927(37.82)$ & 0.074 \\
\hline Cardiac & $14(66.67)$ & $619(69.08)$ & $633(69.03)$ & \\
\hline Non-cardiac & $6(28.57)$ & $217(24.22)$ & $223(24.32)$ & \\
\hline Both & $1(4.76)$ & $60(6.70)$ & $61(6.65)$ & \\
\hline Unknown & 0 & 10 & 10 & \\
\hline Repeat revascularization & $4(12.12)$ & $128(5.54)$ & $132(5.64)$ & 0.104 \\
\hline \multicolumn{5}{|l|}{ 12-month follow-up } \\
\hline Death & $15(31.91)$ & $156(6.54)$ & $171(7.03)$ & $<0.01$ \\
\hline Myocardial Infarction & $2(5.71)$ & $85(3.77)$ & $87(3.80)$ & 0.550 \\
\hline Stroke & $1(3.03)$ & $34(1.51)$ & $35(1.53)$ & 0.481 \\
\hline Re-hospitalization & $22(52.38)$ & $1011(42.50)$ & $1033(42.67)$ & 0.199 \\
\hline Cardiac & 14 (63.64) & $675(67.43)$ & $689(67.35)$ & \\
\hline Non-cardiac & 7 (31.82) & $236(23.58)$ & 243 (23.75) & \\
\hline Both & $1(4.55)$ & 90 (8.99) & $91(8.90)$ & \\
\hline Unknown & 0 & 10 & 10 & \\
\hline Repeat revascularization & 4 (11.76) & $156(6.94)$ & $160(7.01)$ & 0.274 \\
\hline
\end{tabular}

TIMI Thrombolysis in Myocardial Infarction
More studies might be needed to use BARC bleeding definition to clarify the risk of bleeding among different clinical situations. 
Table 4 Multivariable-adjusted odds ratios for the association between in-hospital bleeding and 12 months cardiovascular events

\begin{tabular}{lllll}
\hline Outcome/hazard ratio $(95 \%$ CI $)$ & Unadjusted & Model I & Model II & Model III \\
\hline Primary outcome & & & & \\
Overall cohort & $3.66(2.18-6.17)^{*}$ & $2.85(1.69-4.81)^{*}$ & $1.57(0.91-2.70)$ & $1.42(0.79-2.58)$ \\
STEMI & $2.88(1.35-6.18)^{*}$ & $2.25(1.05-4.84)^{*}$ & $0.98(0.44-2.18)$ & $0.96(0.42-2.17)$ \\
NSTE-ACS & $5.36(2.62-10.95)^{*}$ & $3.82(1.87-7.83)^{*}$ & $2.74(1.29-5.84)^{*}$ & $2.34(0.94-5.86)$ \\
Secondary outcome & & & & $1.43(0.97-2.10)$ \\
Overall cohort & $1.74(1.19-2.53)^{*}$ & $1.63(1.12-2.37)^{*}$ & $1.16(0.69-1.97)$ & $1.02(0.58-1.80)$ \\
STEMI subpopulation & $1.35(0.81-2.25)$ & $1.27(0.76-2.12)$ & $1.95(1.10-3.45)^{*}$ & $1.46(0.73-2.92)$ \\
NSTE-ACS & $2.58(1.49-4.49)^{*}$ & $2.33(1.344 .05)^{*}$ & $1.951)$ \\
\hline
\end{tabular}

Model 1: adjusted for age and sex. Model 2: adjusted for Model 1 covariates + medicine at discharge (aspirin, clopidogrel, angiotensinconverting enzyme inhibitor, angiotensin II receptor blocker, beta-blocker and statin). Model 3: adjusted for Model 2 covariates + creatinine, weight and Killip class

$* p<0.05$

\section{A Product-Limit Survival Estimates - Overall ACS cohort}

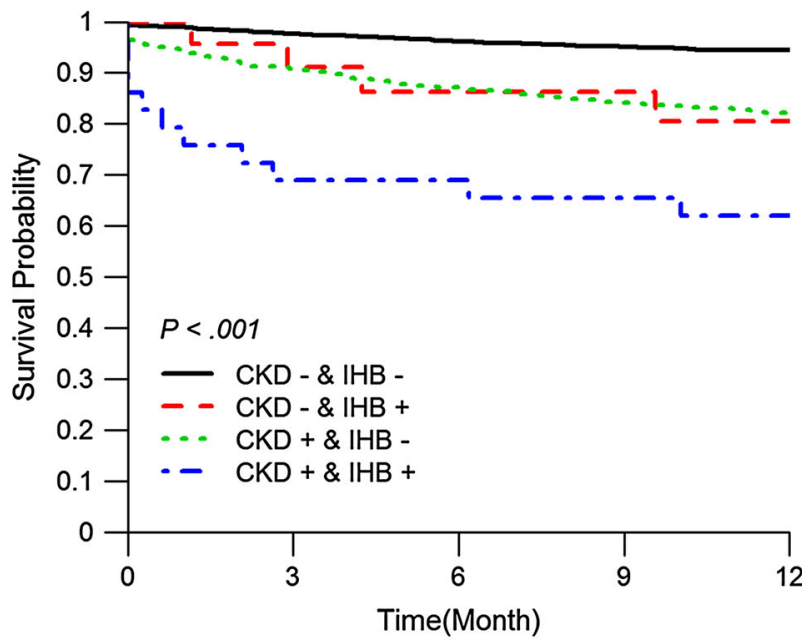

B Product-Limit Survival Estimates - Overall ACS cohort

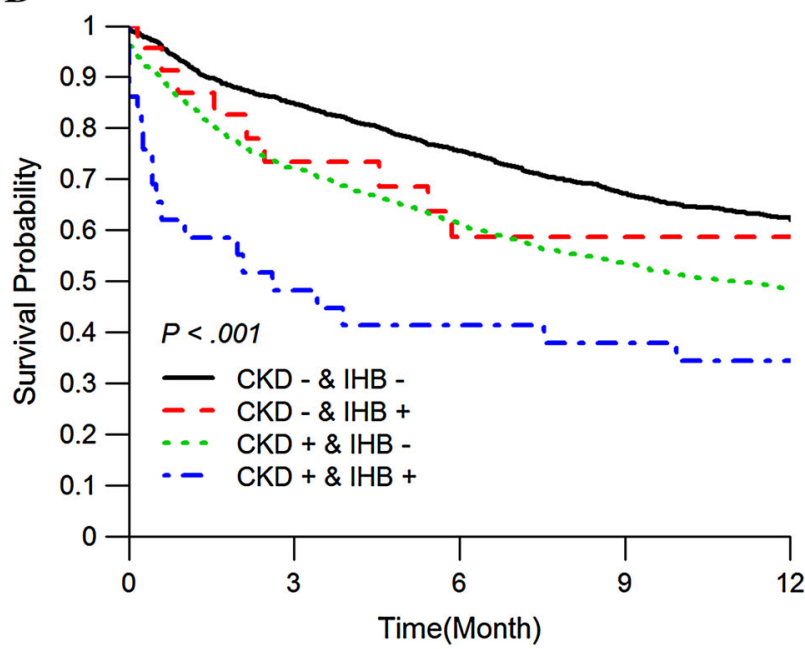

Fig. 1 Kaplan-Meier curve analysis for in-hospital TIMI bleeding and CKD on the a primary and $\mathbf{b}$ secondary end points among the whole populations. TIMI thrombolysis in myocardial infarction, $C K D$ chronic kidney disease, ACS acute coronary syndrome
Table 5 Association between in-hospital bleeding and CKD on primary end point

\begin{tabular}{lllll}
\hline $\begin{array}{l}\text { Groups } \\
n(\%)\end{array}$ & $\begin{array}{l}\text { Primary } \\
\text { end point }(+) \\
(n=274)(\%)\end{array}$ & $\begin{array}{l}\text { Primary end } \\
\text { point }(-) \\
(n=2545)(\%)\end{array}$ & $\begin{array}{l}\text { Adjusted HR } \\
(95 \% \mathrm{CI})^{\mathrm{a}}\end{array}$ & $p$ value \\
\hline $\begin{array}{l}\mathrm{IHB}(-) / \mathrm{CKD} \\
(-)\end{array}$ & $101(36.86)$ & $1745(68.57)$ & 1 & - \\
$\begin{array}{c}\mathrm{IHB}(+) / \mathrm{CKD} \\
(-)\end{array}$ & $5(1.82)$ & $19(0.75)$ & $1.88(0.68-5.21)$ & 0.227 \\
$\begin{array}{c}\mathrm{IHB}(-) / \mathrm{CKD} \\
(+)\end{array}$ & $157(57.30)$ & $763(29.98)$ & $2.13(1.62-2.79)$ & $<0.01$ \\
$\begin{array}{c}\mathrm{IHB}(+) / \mathrm{CKD} \\
(+)\end{array}$ & $11(4.01)$ & $18(0.71)$ & $2.98(1.55-5.75)$ & $<0.01$ \\
\hline
\end{tabular}

$I H B$ in-hospital bleeding, $C K D$ chronic kidney disease

${ }^{a}$ Adjusted for age, sex and medicine at discharge (aspirin, clopidogrel, angiotensin-converting enzyme inhibitor, angiotensin II receptor blocker, betablocker and statin)

Several factors have been reported with IHB such as age, female sex, use of anticoagulation and antiplatelet agents. Different bleeding scores were also developed to calculate the risk of IHB. Mehran et al. used 6 baseline predictors (female sex, age, serum creatinine and white blood cell count, anemia, non-ST-segment elevation MI or ST-segment elevation MI) and 1 treatment-related variable (use of heparin + a glycoprotein IIb/IIIa inhibitor rather than bivalirudin alone) to develop a risk score with c-statistic value 0.74 . Similar to the GUSTO IV-ACS study, our study found IHB were related with glycoprotein IIb/IIIa inhibitor administration. Because there was no definite cardiovascular benefit with adding glycoprotein IIb/IIIa to the standard treatment regimen in Taiwan, we used glycoprotein IIb/IIIa inhibitor limited to the very high cardiovascular risk population $[19,20]$. Our study also found that higher Killip class was related to IHB. The association might just reflect the disease severity and co-morbidity.

In-hospital bleeding is associated with short-, intermediate-, and long-term mortality among patients hospitalized 
for ACS and PCI $[3,4,21]$. Patients with IHB after primary PCI in STE-ACS have significantly increased 3-year rates of morbidity and mortality [22]. The deleterious effect of major bleeding was observed within 1 month, between 1 month and 1 year, and between 1 and 3 years. In patients with NSTE-ACS cumulative mortality was also higher in those who had bleeding vs those without at 30 days, 1 year and 3 years [21]. In our study ACS patients with TIMI bleeding had higher in-hospital and 1-year mortality. Although its causal relationship with mortality is unclear, IHB likely identifies patients with an underlying risk for mortality.

Taiwan has been recognized as an endemic area of kidney disease with the highest incidence and prevalence rates of ESRD in the world [9]. Because patients with CKD have more comorbidity, their treatment strategy in ACS is more complicated in the CKD endemic area. As shown in our study CKD is a poor prognosis factor for those with ACS, possibly because of more extensive and severe atherosclerosis coronary tree with plaque composed of greater necrotic core and less fibrous tissue in the CKD than nonCKD subjects [23-25]. Furthermore, poor antiplatelet responsiveness, underuse of reperfusion therapy, fear of contrast-induced nephropathy during coronary procedure and fewer guideline-recommended treatments prescribed may partly explain why the CKD population had poor prognosis in ACS [26-28].

Renal function impairment is associated with platelet dysfunction and coagulopathy and therefore plays an important role in the risk of bleeding. Creatinine only can be integrated as one risk factor in a clinical score which could identify patients at increased risk for bleeding and subsequent 1-year mortality [29]. Estimated GFR and CKD stages were also related to in-hospital bleeding, cardiovascular events and death [30, 31]. Bleeding itself might further cause renal function deterioration and therefore a vicious cycle develops. In this study we first found inhospital bleeding and CKD might have additively detrimental effect on the cardiovascular outcome. Strategies, such as transradial approach, use of appropriate anticoagulation, antiplatelet therapy and selected use of glycoprotein IIb/IIIa in the high-risk population, to reduce CKD and bleeding are mandatory to reduce subsequent cardiovascular events [32-34].

This study has six main limitations. Firstly, it is a nonrandomized and observational study. Nonetheless, this study provides valuable real-world data on the current practices across the full spectrum of ACS in a CKD endemic area, which could help to improve the ACS management in the CKD population. Second, the mechanism why in-hospital bleeding and CKD have additively detrimental effect of the cardiovascular outcome is unclear. The casual relationship and which one happened first are unknown. Third, the renal end point is not routinely collected after discharge in this registry. Whether those with TIMI bleeding had poor renal outcome is unknown. Fourth, the renal parameter was incorporated into the CRUSADE bleeding risk score, which might be a better score to define bleeding risk. However, we cannot calculate the CRUSADE score because hematocrit was not collected in this registry. Fifth, the prognostic difference between the inhospital hemoglobin changes and TIMI IHB might provide different insight in clinical judgment. However, serial hemoglobin data were not collected during admission in this registry. Sixth, because the interaction test between ACS type and bleeding on outcomes is non-significant, the finding of excess risk in NSTE-ACS was only hypothesis generating.

\section{Conclusion}

In this real-world registry, we found that patients with IHB had higher risk of in-hospital and 12 months death in the ACS population. Furthermore, patients with both IHB and CKD had the worst prognosis during the 12 months followup. Thus, all measures decreasing IHB and preventing CKD in ACS patients are important for eventual cardiovascular risk reduction.

Acknowledgments This study was supported by Sanofi-Aventis Taiwan Co. Ltd. and Bristol-Myers Squibb (Taiwan) Ltd.

We would like to thank the participating physicians and nurses for their contribution in conducting the registry.

Conflict of interest The authors declare that there are no financial or other relationships that could lead to a conflict of interest.

Open Access This article is distributed under the terms of the Creative Commons Attribution License which permits any use, distribution, and reproduction in any medium, provided the original author(s) and the source are credited.

\section{Appendix: ACS Full Spectrum Registry principal investigators}

Kuan-Chen Chang, China University Medical Hospital; Chia-Lin Chao, Taoyuan General Hospital, Department of Health; Yi-Jen Chen, Wan-Fang Hospital; Chien-Cheng Chen, Show Chwan Memorial Hospital; Cheng-Yun Chen, Chia-Yi Christian Hospital; Chung-Yin Chen, Kuang Tien General Hospital; Fu-Tien Chiang, National Taiwan University Hospital; Shao-Yueh Chiang, Cheng Ching Hospital; Li-Ping Chou, Sin Lau Hospital The Presbyterian Church of Taiwan; Ching-Chang Feng, Tainan Municipal Hospital; Charles Jia-Yin Hou, Mackay Memorial Hospital; Kwan-Li Hsu, E-Da Hospital; Tsuei-Yuan Huang, Chi- 
Mei Hospital; Gwo-Ping Jong, Taichung Armed Forces General Hospital; Yu-Lin Ko, Taipei Tzu Chi General Hospital; Wen-Ter Lai, Kaohsiung Medical University Chung-Ho Memorial Hospital; Wen-Lieng Lee, Taichung Veterans General Hospital; Chun-I Lee, Pingtung Christian Hospital; Meng-Huan Lei, Lo-Tung Po-Ai Hospital; AiHsien Li, Far Eastern Memorial Hospital; Yi-Heng Li, National Cheng Kung University Hospital; Jou-Wei Lin, National Taiwan University Hospital, Yun-lin Branch; TinKwang Lin, Dalin Tzuchi General Hospital; Jih-Min Lin, Kee-lung Hospital, Department of Health; Shing-Jong Lin, Taipei Veterans General Hospital; Hung-Shun Lo, Cathay General Hospital; Guang-Yuan Mar, Kaohsiung Veterans General Hospital; Chun-Ming Shih, Taipei Medical University Hospital; Kou-Gi Shyu, Shin Kong Wu Ho-Su Memorial Hospital; Cheng-Dao Tsai, Changhua Christian Hospital; Chuen-Den Tseng, National Taiwan University Hospital; Kwo-Chang Ueng, Chung Shan Medical University Hospital; Ji-Hung Wang, Hualien Tzu Chi General Hospital; Kuang-Te Wang, Mackay Memorial Hospital, Taitung Branch; Ming-Shien Wen, Linkou Chang Gung Memorial Hospital; Szu-Chi Wen, Hsin Chu General Hospital, Department of Health; Chiung-Jen Wu, Kaohsiung Chang Gung Memorial Hospital; Shih-Peng Yang, Tri-Service General Hospital; Wei-Hsian Yin, Cheng-Hsin Hospital.

\section{References}

1. Nordlie MA, Wold LE, Kloner RA (2005) Genetic contributors toward increased risk for ischemic heart disease. J Mol Cell Cardiol 39(4):667-679

2. Taiwan Department of Health. Statistics data statistical analysis of 2009 mortality data. Press conference on 2009 mortality data. http://www.doh.gov.tw. Accessed 19 Jan 2011

3. Maréchaux S, Barrailler S, Pinçon C, Decourcelle V, Guidez T, Braun S, Bouabdallaoui N, Bauchart JJ, Auffray JL, Juthier F, Banfi C, Susen S, Jude B, Asseman P, Van Belle E, Ennezat PV (2012) Prognostic value of hemoglobin decline over the GRACE score in patients hospitalized for an acute coronary syndrome. Heart Vessel 27(2):119-127

4. Lindsey JB, Marso SP, Pencina M, Stolker JM, Kennedy KF, Rihal C, Barsness G, Piana RN, Goldberg SL, Cutlip DE, Kleiman NS, Cohen DJ, EVENT Registry Investigators (2009) Prognostic importance of bleeding and myocardial infarction after percutaneous coronary intervention in unselected patients. JACC Interv 2:1074-1082

5. Wiviott SD, Braunwald E, McCabe CH, Montalescot G, Ruzyllo W, Gottlieb S, Neumann FJ, Ardissino D, De Servi S, Murphy SA, Riesmeyer J, Weerakkody G, Gibson CM, Antman EM, TRITON-TIMI 38 Investigators (2007) Prasugrel versus clopidogrel in patients with acute coronary syndromes. N Engl J Med 357(20):2001-2015

6. Melloni C, Alexander KP, Chen AY, Newby LK, Roe MT, Allen LaPointe NM, Pollack CV Jr, Gibler WB, Ohman EM, Peterson ED, CRUSADE Investigators (2008) Unfractionated heparin dosing and risk of major bleeding in non-ST-segment elevation acute coronary syndromes. Am Heart J 56(2):209-215

7. Chronic Kidney Disease Prognosis Consortium, Matsushita K, van der Velde M, Astor BC, Woodward M, Levey AS, de Jong PE, Coresh J, Gansevoort RT (2010) Association of estimated glomerular filtration rate and albuminuria with all-cause and cardiovascular mortality in general population cohorts: a collaborative meta-analysis. Lancet 375(9731):2073-2081

8. Matsue Y, Matsumura A, Abe M, Ono M, Seya M, Nakamura T, Iwatsuka R, Mizukami A, Setoguchi M, Nagahori W, Ohno M, Suzuki M, Hashimoto Y (2013) Prognostic implications of chronic kidney disease and anemia after percutaneous coronary intervention in acute myocardial infarction patients. Heart Vessel 28(1):19-26

9. USRDS (2009) International comparisons. In: United Stated Renal Data System Annual Data Report. The National Institute of health, National Institute of diabetes and digestive and kidney disease. Bethesda, pp 344-355

10. Shyu KG, Wu CJ, Mar GY, Hou Charles JY, Li AH, Wen MS, Lai WT, Lin SJ, Kuo ChT, Hwang JJ, Chiang FT (2011) Clinical characteristics, management and in-hospital outcomes of patients with acute coronary syndrome-observations from the Taiwan ACS Full Spectrum Registry. Acta Cardiol Sin 27:135-144

11. International Epidemiological Association guidelines. http:// www.ieaweb.org/index.php?option=com_content\&view=arti cle\&id $=15 \% 3$ Agood-epidemiological-practice-gep\&catid $=20 \%$ 3 Agood-epidemiological-practice-gep $\&$ Itemid $=43 \&$ limitstart $=1$. Accessed 28 Jan 2011

12. Chesebro JH, Knatterud G, Roberts R, Borer J, Cohen LS, Dalen J, Dodge HT, Francis CK, Hillis D, Ludbrook P (1987) Thrombolysis in myocardial infarction (TIMI) trial, phase I: a comparison between intravenous tissue plasminogen activator and intravenous streptokinase. Clinical findings through hospital discharge. Circulation 76(1):142-154

13. Levey AS, Greene T, Kusek JW (2000) A simplified equation to predict glomerular filtration rate from serum creatinine. J Am Soc Nephrol 11:A0828

14. National Kidney Foundation (2002) K/DOQI clinical practice guidelines for chronic kidney disease: evaluation, classification, and stratification. Am J Kidney Dis 39((2 Suppl 1)):S1-S266

15. Valente S, Lazzeri C, Chiostri M, Osmanagaj L, Giglioli C, Gensini GF (2011) STEMI patients-the more you bleed, the more you die: a comparison between classifications. Clin Cardiol 34(2):90-96

16. Rao SV, O'Grady K, Pieper KS, Granger CB, Newby LK, Mahaffey KW, Moliterno DJ, Lincoff AM, Armstrong PW, Van de Werf F, Califf RM, Harrington RA (2006) A comparison of the clinical impact of bleeding measured by two different classifications among patients with acute coronary syndromes. J Am Coll Cardiol 47(4):809-816

17. Mehran R, Rao SV, Bhatt DL, Gibson CM, Caixeta A, Eikelboom J, Kaul S, Wiviott SD, Menon V, Nikolsky E, Serebruany V, Valgimigli M, Vranckx P, Taggart D, Sabik JF, Cutlip DE, Krucoff MW, Ohman EM, Steg PG, White H (2011) Standardized bleeding definitions for cardiovascular clinical trials: a consensus report from the Bleeding Academic Research Consortium. Circulation 123:2736-2747

18. Ndrepepa G, Schuster T, Hadamitzky M, Byrne RA, Mehilli J, Neumann FJ, Richardt G, Schulz S, Laugwitz KL, Massberg S, Schömig A, Kastrati A (2012) Validation of the Bleeding Academic Research Consortium definition of bleeding in patients with coronary artery disease undergoing percutaneous coronary intervention. Circulation 125(11):1424-1431

19. Yip HK, Wu CJ, Chang HW, Hsieh YK, Fang CY, Chen SM, Chen MC (2003) Impact of tirofiban on angiographic morphologic features of high-burden thrombus formation during direct 
percutaneous coronary intervention and short-term outcomes. Chest 124(3):962-968

20. Li YH, Yeh HI, Tsai CT, Liu PY, Lin TH, Wu TC, Hung KC, Hsieh YC, Mar GY, Fang CY, Chiu KM, Chen JH (2012) 2012 Guidelines of the Taiwan Society of Cardiology (TSOC) for the management of ST-Segment Elevation Myocardial Infarction. Acta Cardiol Sin 28:63-89

21. Lopes RD, Subherwal S, Holmes DN, Thomas L, Wang TY, Rao SV, Magnus Ohman E, Roe MT, Peterson ED, Alexander KP (2012) The association of in-hospital major bleeding with short-, intermediate-, and long-term mortality among older patients with non-ST-segment elevation myocardial infarction. Eur Heart J 33(16):2044-2053

22. Suh JW, Mehran R, Claessen BE, Xu K, Baber U, Dangas G, Parise H, Lansky AJ, Witzenbichler B, Grines CL, Guagliumi G, Kornowski R, Wöhrle J, Dudek D, Weisz G, Stone GW (2011) Impact of in-hospital major bleeding on late clinical outcomes after primary percutaneous coronary intervention in acute myocardial infarction the HORIZONS-AMI (Harmonizing Outcomes with Revascularization and Stents in Acute Myocardial Infarction) trial. J Am Coll Cardiol 58(17):1750-1756

23. Morel O, El Ghannudi S, Jesel L, Radulescu B, Meyer N, Wiesel ML, Caillard S, Campia U, Moulin B, Gachet C, Ohlmann P (2011) Cardiovascular mortality in chronic kidney disease patients undergoing percutaneous coronary intervention is mainly related to impaired P2Y12 inhibition by clopidogrel. J Am Coll Cardiol 57(4):399-408

24. Keough-Ryan TM, Kiberd BA, Dipchand CS, Cox JL, Rose CL, Thompson KJ, Clase CM (2005) Outcomes of acute coronary syndrome in a large Canadian cohort: impact of chronic renal insufficiency, cardiac interventions, and anemia. Am J Kidney Dis 46:845-855

25. Masoudi FA, Plomondon ME, Magid DJ, Sales A, Rumsfeld JS (2004) Renal insufficiency and mortality from acute coronary syndromes. Am Heart J 147:623-629

26. Baber U, Stone GW, Weisz G, Moreno P, Dangas G, Maehara A, Mintz GS, Cristea E, Fahy M, Xu K, Lansky AJ, Wennerblom B, Mathey DG, Templin B, Zhang Z, Serruys PW, Mehran R (2012) Coronary plaque composition, morphology, and outcomes in patients with and without chronic kidney disease presenting with acute coronary syndromes. JACC Cardiovasc Imaging 5(3 Suppl):S53-S61

27. El-Menyar A, Zubaid M, Sulaiman K, Singh R, Al Thani H, Akbar M, Bulbanat B, Al-Hamdan R, Almahmmed W, Al Suwaidi J (2010) In-hospital major clinical outcomes in patients with chronic renal insufficiency presenting with acute coronary syndrome: data from a Registry of 8176 patients. Mayo Clin Proc 85(4):332-340

28. Undas A, Nycz K, Pastuszczak M, Stompor T, Zmudka K (2010) The effect of chronic kidney disease on fibrin clot properties in patients with acute coronary syndrome. Blood Coagul Fibrinolysis 21(6):522-527

29. Mehran R, Pocock SJ, Nikolsky E, Clayton T, Dangas GD, Kirtane AJ, Parise H, Fahy M, Manoukian SV, Feit F, Ohman ME, Witzenbichler B, Guagliumi G, Lansky AJ, Stone GW (2010) A risk score to predict bleeding in patients with acute coronary syndromes. J Am Coll Cardiol 55(23):2556-2566

30. Attallah N, Yassine L, Fisher K, Yee J (2005) Risk of bleeding and restenosis among chronic kidney disease patients undergoing percutaneous coronary intervention. Clin Nephrol 64(6):412-418

31. Fox CS, Muntner P, Chen AY, Alexander KP, Roe MT, Cannon CP, Saucedo JF, Kontos MC, Wiviott SD, Acute Coronary Treatment and Intervention Outcomes Network registry (2010) Use of evidence-based therapies in short-term outcomes of STsegment elevation myocardial infarction and non-ST-segment elevation myocardial infarction in patients with chronic kidney disease: a report from the National Cardiovascular Data Acute Coronary Treatment and Intervention Outcomes Network registry. Circulation 121(3):357-365

32. Cantor WJ, Mahaffey KW, Huang Z, Das P, Gulba DC, Glezer S, Gallo R, Ducas J, Cohen M, Antman EM, Langer A, Kleiman NS, White HD, Chisholm RJ, Harrington RA, Ferguson JJ, Califf RM, Goodman SG (2007) Bleeding complications in patients with acute coronary syndrome undergoing early invasive management can be reduced with radial access, smaller sheath sizes, and timely sheath removal. Catheter Cardiovasc Interv 69(1):73-83

33. Mehran R, Lansky AJ, Witzenbichler B, Guagliumi G, Peruga JZ, Brodie BR, Dudek D, Kornowski R, Hartmann F, Gersh BJ, Pocock SJ, Wong SC, Nikolsky E, Gambone L, Vandertie L, Parise H, Dangas GD, Stone GW, HORIZONS-AMI Trial Investigators (2009) Bivalirudin in patients undergoing primary angioplasty for acute myocardial infarction (HORIZONS-AMI): 1-year results of a randomised controlled trial. Lancet 374(9696):1149-1159

34. Becker RC, Bassand JP, Budaj A, Wojdyla DM, James SK, Cornel JH, French J, Held C, Horrow J, Husted S, Lopez-Sendon J, Lassila R, Mahaffey KW, Storey RF, Harrington RA, Wallentin L (2011) Bleeding complications with the P2Y12 receptor antagonists clopidogrel and ticagrelor in the PLATelet inhibition and patient Outcomes (PLATO) trial. Eur Heart J 32(23):2933-2944 